\title{
París, California y la búsqueda por una teoría del cambio cultural
}

\author{
Paris, California and the quest for a theory of cultural change
}

\author{
Lorenzo Baravalle \\ Centro de Ciências Naturais e Humanas, Universidade Federal do ABC, Brasil \\ Centro de Filosofia das Ciências, Universidade de Lisboa, Portugal \\ lorenzo_baravalle@yahoo.it
}

\begin{abstract}
Resumen
El debate sobre la posibilidad de una teoría evolutiva del cambio cultural se ha acalorado, a lo largo de los últimos años, debido a las supuestas incompatibilidades entre dos de las principales propuestas teóricas en ese ámbito disciplinar: la teoría de la doble herencia (o de la herencia dual) y la epidemiología cultural. La primera, formulada a partir de los años 1980 por un grupo de biólogos y antropólogos situados principalmente en universidades californianas, defiende una analogía entre herencia genética y transmisión cultural. La epidemiología cultural, más recientemente formulada por Dan Sperber y sus colaboradores (situados principalmente en universidades parisinas), niega que tal analogía sea sostenible y propone un modelo parcialmente alternativo. Pero ¿cuánto, en realidad, estas dos propuestas difieren entre sí? En este artículo defenderé que menos de lo que los epidemiólogos culturales suelen sostener.
\end{abstract}

Palabras clave: evolución cultural, genética de poblaciones, epidemiología, atractores culturales, niveles de causación.

\begin{abstract}
The debate on the possibility of an evolutionary theory of cultural change has heated up, over the last years, due to the supposed incompatibilities between the two main theoretical proposals in the field: dual inheritance theory and cultural epidemiology. The former, first formulated in the 1980's by a group of biologists and anthropologists mostly hosted at Californian universities, supports an analogy between genetic inheritance and cultural transmission. Cultural epidemiology, more recently formulated by Dan Sperber and his
\end{abstract}

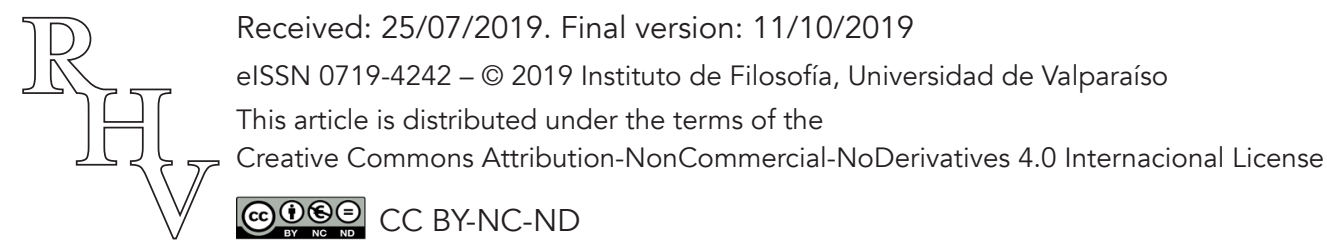


collaborator (mostly hosted at Parisian universities), denies the defensibility of such an analogy and put forward a partially alternative model. But how much do these proposals actually differ with each other? In this article, I shall argue that less than what cultural epidemiologists use to think.

Keywords: cultural evolution, population genetics, epidemiology, cultural attractors, levels of causation.

\section{Introducción}

En el contexto de los estudios sobre evolución cultural, dos propuestas teóricas han estado compitiendo para asegurarse un papel predominante en los futuros desarrollos de la disciplina: la teoría de la doble herencia (o de la herencia dual; Boyd y Richerson 1985) y la epidemiología cultural (Sperber 1996). El desacuerdo entre los sostenedores de las dos teorías se ha ido configurando, a lo largo de los últimos veinte años, aproximadamente de la siguiente manera. Por un lado, los epidemiólogos culturales han criticado la teoría de la doble herencia por presentar una imagen de los procesos de cambio cultural poco realista. Por el otro, los simpatizantes de la teoría de la doble herencia no descartan la epidemiología cultural como incorrecta, sino que la consideran compatible con la teoría de la doble herencia e insisten sobre la necesidad de integrarla a esta.

Aunque la discusión entre los "californianos" (los teóricos de la doble herencia) y los "parisinos" (los epidemiólogos culturales; según la denominación geográfica de Sterelny 2017) haya generado útiles aclaraciones sobre algunas de las tesis centrales de cada escuela (Richerson y Boyd 2005; Sperber y Claidière 2008; Acerbi y Mesoudi 2015; Morin 2016a), actualmente parece haber llegado a un estado de impasse. Entre los temas de desacuerdo, el que sobre más énfasis ha sido puesta por parte de los epidemiólogos cultural es el de la fidelidad de la transmisión de la información cultural (véase, para una discusión reciente, Morin 2016b). Mientras que para los californianos tal proceso, si bien menos fiel que la herencia genética, es suficientemente estable como para garantizar, a lo largo de las generaciones, la preservación de la información cultural, para los parisinos las cosas son más complicadas que eso. Durante cada instancia de comunicación, los sujetos reinterpretan la información cultural de manera tan idiosincrásica que no tiene sentido identificar alguna entidad que perdure en ese proceso de manera estable.

Claramente, los parisinos concuerdan sobre el hecho de que las culturas humanas exhiben acumulación de hábitos, tradiciones y técnicas. Sin embargo, a diferencia de los californianos, niegan que tal fenómeno pueda ser "substancializado" de manera análoga al proceso de herencia genética. Una sociedad no es, en última instancia, nada más que un conjunto de sujetos interactuando entre ellos y con el ambiente: lo que llamamos "acumulación cultural" es un epifenómeno resultante de tales interacciones. Los 
epidemiólogos culturales creen que esta es una explicación de las dinámicas culturales más naturalísticamente aceptable que la de los teóricos de la doble herencia (Scott-Phillips et al. 2018). Los teóricos de la doble herencia, por otro lado, se rehúsan a considerar tal propuesta como totalmente distinta de la de ellos mismos (Richerson 2017).

En este artículo, mi objetivo es ofrecer algunas consideraciones que contribuyan a entender mejor los términos de la controversia $y$, tal vez, indicar una manera para solucionarla. Por lo general, creo que los teóricos de la doble herencia estén en lo cierto. En mi opinión, la epidemiología cultural ofrece una caracterización de los procesos cognitivos involucrados en la comunicación humana que es, efectivamente, diferente de aquella de la teoría de la doble herencia (y puede que incluso mejor que esta). Sin embargo, no me parece que, en la práctica, tal caracterización deba llevarnos a considerar las dinámicas de acumulación y cambio cultural de manera diferente de como son representadas en el contexto de la teoría de la doble herencia.

El plan del artículo es el siguiente. En la segunda sección introduzco los que considero ser los dos explananda centrales de cualquier teoría de la evolución cultural y discuto las explicaciones proporcionadas, respectivamente, por la teoría de la doble herencia y la epidemiología cultural a cada uno de ellos. En la tercera sección intento entender en que aspectos los parisinos advierten mayor incompatibilidad entre su propia propuesta y la teoría de la doble herencia. En esta misma sección, digo algo más sobre el por qué los parisinos se autodenominan "epidemiólogos" y discuto supuestas diferencias entre los modelos empleados por ellos y los modelos empleados por los californianos, de alguna manera derivados de la genética de poblaciones. En la sección 4 argumento que, de un punto de vista formal, los modelos empleados por la epidemiología cultural y la teoría de la doble herencia son compatibles y complementarios. A partir de este resultado, muestro en la sección 5 que, si bien las quejas de los parisinos sobre de la teoría de la doble herencia puedan tener un cierto valor filosófico, ellas no tienen la relevancia científica que le suele ser atribuida. En la conclusión, resumo brevemente mi argumento, sus premisas y sus implicaciones.

\section{Lo que una teoría de la evolución cultural debería explicar}

Tanto la epidemiología cultural como la teoría de la doble herencia se presentan como teorías de la evolución cultural. Para entender mejor las diferencias entre las dos propuestas es, por lo tanto, provechoso preguntarse cuales son sus objetivos compartidos.

Aunque no sea un rasgo necesario de todas las teorías de la evolución cultural, la mayoría de ellas (o, por lo menos, las que han ido apareciendo a lo largo de los últimos 3040 años) tiende a considerar la cultura (principalmente humana, pero no necesariamente) tanto como un producto de la evolución biológica como un proceso evolutivo parcialmente independiente. Los dos fenómenos están relacionados; sin embargo, 
merecen consideraciones distintas. Como veremos más adelante, uno de los aspectos más importantes - pero también frecuentemente ignorado - del desacuerdo entre parisinos y californianos concierne en que medida consideraciones sobre la evolución de nuestras habilidades cognitivas deberían condicionar consideraciones sobre el cambio cultural. De momento, no obstante, vamos a concentrarnos sobre los dos fenómenos por separado.

Explicar la cultura como producto de la evolución biológica significa explicitar las condiciones bajo las cuales facultades cognitivas responsables por el aprendizaje social, la cooperación y la comunicación han evolucionado. También significa identificar, en la medida de lo posible, en que modo la evolución de nuestras facultades cognitivas condiciona la manera en la que, hoy en día, aprendemos información cultural de los demás y emulamos conductas ajenas de manera selectiva.

Con relación a este elemento de la teoría de la evolución cultural, los parisinos y los californianos, aunque en desacuerdo sobre aspectos específicos (Sperber y Claidière 2008), comparten varias tesis generales. Ambas escuelas se inspiran en la psicología evolucionista y, a la vez, se distancian de esta por razones similares. De la psicología evolucionista, parisinos y californianos adoptan la idea de que buena parte de nuestra psicología social evolucionó durante la era geológica conocida como Pleistoceno (entre 2,59 millones y 10 mil años atrás). Sin embargo, tanto los parisinos como los californianos tienen una concepción diferente de los psicólogos evolucionistas con relación al tipo de habilidades cognitivas que fueron seleccionadas durante esa época.

Mientras los psicólogos evolucionistas (por lo menos los más tradicionales) piensan que lo que evolucionó en el Pleistoceno fueron una serie de módulos cognitivos para tareas relativamente específicas (fabricación de herramientas, vida en grupo, relaciones estables de pareja etc.), los evolucionistas culturales creen que la selección natural premió, durante ese periodo, habilidades más genéricas relacionadas con la transmisión de la información aprendida. Estas diferentes concepciones fundamentan dos distintas interpretaciones de la cultura. Para los psicólogos evolucionistas (Tooby y Cosmides 1992), nuestros hábitos y tradiciones eran adaptativos en la época en la que los módulos cognitivos que permiten implementarlos fueron seleccionados; hoy en día, ellos son mayoritariamente disfuncionales. Para los evolucionistas culturales (tanto parisinos como californianos) la cultura es - en virtud del hecho de que nuestras habilidades cognitivas son más genéricas y flexibles - capaz de cambiar a lo largo del tiempo y, aunque a veces nuestros hábitos y tradiciones sean efectivamente disfuncionales, no deja de ser globalmente adaptativa.

Boyd y Richerson (1985; Richerson y Boyd 2005) identifican tres tipos de sesgos (biases) relacionados con el aprendizaje social: sesgos dependientes del contenido, conformismo, y sesgos dependientes del modelo (estos últimos dos son a veces denominados "sesgos dependientes del contexto"). La idea básica es que una cierta composición de creencias, hábitos, tradiciones y técnicas en una sociedad es el producto del hecho de que las personas tienden a adoptar, por razones evolutivas biológicas, actitudes y conductas que son: 
- atractivas de acuerdo con algún criterio psicológico, o cuya utilidad es evidente, o simples de entender (sesgos dependientes del contenido);

- adoptadas por la mayoría de los miembros de la población (conformismo);

- adoptadas por los miembros más prestigiosos de la población(sesgo dependiente del modelo).

Cada uno de estos sesgos es tendencialmente adaptativo, de un punto de vista biológico/genético, aunque ninguno es infalible, y puede fácilmente pasar que — como consecuencia de repetidas instancias de aprendizaje social conforme estos sesgos- en una población se difundan actitudes y conductas no adaptativas, o incluso maladaptativas (esto es, negativas del punto de vista de la fitness genética).

Los epidemiólogos culturales no tienen una propuesta tan detallada con respecto a los tipos de sesgos que actuarían en la transmisión de información aprendida. En los textos clásicos de los autores pertenecientes a esa tradición (en particular, Sperber 1996; Sperber y Claidière 2007), se puede percibir un énfasis sobre lo que Boyd y Richerson llaman "sesgos dependientes del contenido". De acuerdo con la posición defendida en textos más recientes (Claidière et al. 2014; Buskell 2017a), parecería que también sesgos más "contextuales" pueden condicionar la difusión de un hábito o una tradición. De cualquier manera, aquí voy simplemente a asumir que hay compatibilidad y solamente un desacuerdo menor entre parisinos y californianos. Probablemente, estrictamente hablando, la propuesta de Boyd y Richerson es aún algo programática, y ulteriores estudios necesitan ser realizados para corroborar o ampliar la taxonomía de sesgos que acabo de exponer. Sin embargo, ese trabajo ya está siendo realizado (véase Kendal et al. 2018 para una reseña actualizada), y los resultados son aparentemente coherentes tanto con la imagen defendida por los parisinos como aquella defendida por los californianos.

Las diferencias entre los epidemiólogos culturales y los teóricos de la doble herencia se hacen más evidentes con relación a la caracterización de la cultura como un proceso evolutivo en si mismo. Discutiendo la cultura como producto evolutivo, nos hemos concentrado sobre la dimensión psicológica/cognitiva del fenómeno cultural, con un énfasis sobre los mecanismos que hacen que un individuo adopte una actitud o una conducta en lugar que otra. Cuando una teoría de la evolución cultural pasa a considerar la cultura como un proceso evolutivo, el foco deja de ser el individuo y pasa a ser la población, o sociedad, de la cual el individuo forma parte. Lo que una teoría de la evolución cultural como teoría de los procesos de acumulación y cambio cultural pretende explicar es por qué, en una determinada población o sociedad, ciertas creencias, hábitos, tradiciones, técnicas etc. gozan de mayor difusión que otros.

Para los sostenedores de la teoría de la doble herencia, el fenómeno de difusión de creencias, hábitos, tradiciones, técnicas etc. debe ser concebido en estricta analogía con los fenómenos de evolución fenotípica. De hecho, podemos considerar las actitudes y las 
conductas derivadas de la adopción de ciertas creencias o tradiciones culturales como un subconjunto de rasgos fenotípicos, los cuales tienen, justamente, la peculiaridad de poder ser transmitidos por comunicación y aprendizaje social. Boyd y Richerson llaman tales rasgos fenotípicos "variantes culturales". Del punto de vista de la transmisión de las variantes culturales, el aprendizaje social funciona como si fuera un canal paralelo a la herencia genética. Es justamente en virtud de esta analogía que los californianos denominan a su propia teoría de teoría de la "doble herencia".

Obviamente, la transmisión cultural tiene diferencias significativas con respecto a la herencia genética. En particular, la herencia genética es principalmente vertical - la información genética es transmitida de padres a hijos (aunque transferencias de información genética horizontal sean comunes en bacterias) - mientras que la información cultural fluye en todas las direcciones (de padres a hijos, entre padres, entre hijos, entre adultos de la generación de los padres a niños que no son sus hijos, de hijos a padres etc.). Aún admitiendo estas diferencias, los sostenedores de la teoría de la doble herencia creen que es posible aplicar una serie de modelos matemáticos derivados de la genética de poblaciones - $\mathrm{y}$, más específicamente, de la genética cuantitativa - para determinar los factores que, en casos concretos, influyeron en una particular distribución de variantes culturales en una población. Tales factores son los factores evolutivos tradicionales selección natural, deriva genética, migración etc.--, más otros análogos, pero típicamente culturales, como la mutación dirigida (innovación cultural), factores de transformación de la variante, o diferentes tipos de selección cultural (Mesoudi 2011; Baravalle 2019).

Los epidemiólogos culturales, por otro lado, ven las diferencias entre transmisión cultural y herencia genética como fatales para la posible aplicación de modelos derivados de la genética de poblaciones a la difusión de ítems culturales. Eso los lleva a rechazar varios aspectos de la conceptualización de Boyd y Richerson, como veremos en más detalle en la próxima sección.

\section{Epidemiología y atractores culturales}

Para los epidemiólogos culturales, la transmisión cultural no es análoga a la herencia genética porque - con la excepción de unos casos específicos - la información cultural no se replica, simplemente, de individuo a individuo, sino que es continuamente reformulada (Sperber 1996; 2001). Esto es, cuando alguien comunica una información potencialmente transmisible (en el sentido de que podría influenciar la actitud o la conducta del interlocutor) es muy poco probable que lo haga de manera que la representación que tiene de tal información sea transmitida fielmente al interlocutor. El aprendizaje de información cultural involucra una constante reconceptualización, por parte del aprendiz, de la información aprendida. Tal reconceptualización es debida al hecho de que el aprendiz entra en contacto con la información cultural con su propio bagaje de conocimientos anteriores, habilidades inferenciales e intereses específicos. Por

Revista de Humanidades de Valparaíso, 2019, No 14, 223-240 
esta razón, la representación que dos personas elegidas al azar en una población tienen de un mismo ítem cultural (que sea un elemento fonético del lenguaje, un ritual religioso, una técnica de preparo de un alimento o una creencia científica) tiende a ser siempre levemente diferente e idiosincrásica.

Pero ¿como explicar, entonces, el hecho de que existen hábitos y tradiciones compartidas entre miembros de una sociedad, las cuales parecen perdurar a lo largo de las generaciones? Para ofrecer una respuesta a esta pregunta, los parisinos invocan la analogía entre procesos de difusión cultural y los procesos epidemiológicos. Los miembros de una sociedad son portadores de representaciones culturales, de manera similar a como los enfermos son portadores de una enfermedad. En sus interacciones diarias, los dos tienen una cierta probabilidad de transmitir los "gérmenes" de la representación o de la enfermedad. Cuando un nuevo sujeto es infectado, desarrolla su propia versión de la representación o de la enfermedad. Esta puede - y suele, como acabamos de ver- tener alguna diferencia respecto a la versión (o las versiones) de los individuos con los que entró en contacto. Sin embargo, tales diferencias no pueden superar un cierto umbral; si lo hacen, la nueva representación no es, estrictamente hablando, la "misma" representación o enfermedad. No podemos decir que alguien contrajo, efectivamente, el sarampión si de hecho carece de todos los síntomas característicos de esta enfermedad. De la misma manera, no podemos decir que alguien se convirtió realmente al Zoroastrismo si no exhibe una conducta coherente con la de esta religión.

Hay que aclarar en seguida, ya que es crucial para entender la perspectiva de los epidemiólogos culturales, que no hay nada de intrínseco a la representación cultural que impida que ella varíe incluso radicalmente a lo largo de los "contagios". Lo que hace que ella se mantenga relativamente estable en una población es el hecho de que los miembros de la población dejen de considerar representaciones aberrantes como posibles instancias de la representación original. Esta idea puede ser expresada más rigurosamente por medio de la noción de atractor. Un atractor es un área, en un espacio ideal de posibles variaciones subjetivas de una representación cultural, en el que las representaciones reales tienden a converger (véase Buskell 2017a y Scott-Phillips et al. 2018 para análisis detallados de este concepto).

Imagínese, por ejemplo, el espacio de posibles variaciones de la letra zeta en castellano. Como es sabido, en la mayoría del territorio español, la letra es pronunciada aproximadamente como la th inglesa, mientras que en Latinoamérica es comúnmente pronunciada como una $s$. Pequeñas variaciones (como, por ejemplo, en dirección al fonema $/ d z /$ ) son toleradas y entendidas. Sin embargo, un individuo que pronunciara la letra zeta de una manera radicalmente distinta (como una $k a$, por ejemplo) tendría serios problemas de comunicación. Eso puede ser expresado en términos de atracción cultural, con el auxilio de la figura 1, de la siguiente manera. Las zonas más obscuras del espacio de variación representan los fonemas correctos, los cuales, junto con los fonemas representados por las zonas grises representan los fonemas aceptados. Las zonas blancas

Revista de Humanidades de Valparaíso, 2019, No 14, 223-240 
representan los fonemas incorrectos. La representación cultural se transmite solo en los casos en los que el nuevo hablante sea capaz de elaborar una conducta (en este caso lingüística) correspondiente a la de las áreas más obscuras o grises.

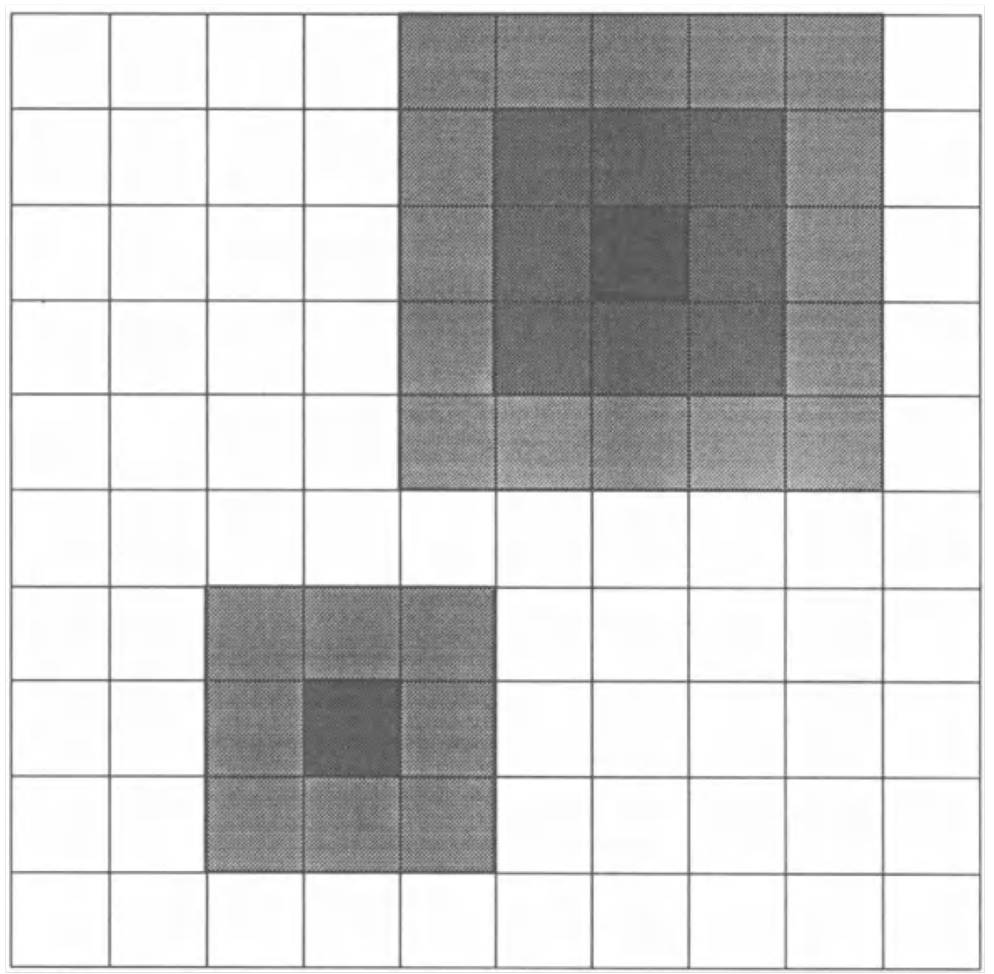

Fig. 1: Reconstrucción de un espacio de variación cultural con dos atractores (de Sperber 1996, 109).

Los atractores de una determinada representación pueden cambiar gradualmente a lo largo del tiempo, tanto por razones relacionadas con la adaptividad biológica de las conductas relacionadas con la representación, como de otro tipo (por sesgos "de contenido", contextuales u otros). El aspecto más importante a resaltar aquí es que, a diferencia del canal de herencia cultural postulado por los teóricos de la doble herencia, la noción de atractor cultural no tiene ninguna relevancia causal:

Un atractor, por como lo he caracterizado, es una construcción abstracta, estadística, como la tasa de mutación o la probabilidad de transformación. Decir que hay un atractor sólo quiere decir que, en un determinado espacio de posibilidades, las probabilidades de transformación forman un cierto patrón [...] (Sperber 1996, 111-112)

El espacio de variación no es, pues, nada más que un artefacto estadístico útil para representar la permanencia o el cambio de determinadas tradiciones o costumbres. 
El error de los teóricos de la doble herencia, desde esta perspectiva, sería el de por así decirlo_ "hipostasiar" el proceso de cambio cultural (Sperber 2001; Claidière et al. 2014): es incorrecto hablar de un "canal" de transmisión cultural porque en última instancia no hay nada, en la cultura, más allá que un conjunto de personas interactuando entre sí y con el ambiente de cierta manera. A diferencia de la analogía con la herencia genética, engañosa y simplista (porque reduce el proceso de transmisión cultural a mera replicación, ocultando la complejidad de las relaciones sociales) la analogía con la epidemiología sería más refinada y realista. Esta capturaría aspectos de la emergencia de patrones poblacionales a partir de agregados de interacciones individuales de manera que quedaría inaccesible a una perspectiva fundamentada en la analogía con la genética de poblaciones (Buskell 2017b).

\section{Epidemiología y genética de poblaciones: una comparación}

A partir de esta sección, voy a evaluar si los epidemiólogos culturales tienen razones válidas para justificar la realización de un programa de investigación significativamente distinto de aquel iniciado por los teóricos de la doble herencia. Procederé, aproximadamente, de la siguiente manera. En esta sección compararé algunos aspectos de la genética de poblaciones y de la epidemiología para averiguar si, como asumido por los epidemiólogos culturales, las analogías con estas dos disciplinas conducen a perspectivas genuinamente alternativas sobre los fenómenos culturales. Como veremos, no hay razones para pensar que ese sea el caso. En la próxima sección, entonces, consideraré una posible respuesta de los epidemiólogos culturales. Aunque la genética de poblaciones y la epidemiología sean, bajo muchos puntos de vista, equivalentes, se podría argumentar que el hecho de ver el fenómeno de cambio cultural como un proceso dinámico causalmente autónomo o, alternativamente, como meramente resultante de agregados de interacciones individuales debe hacer alguna diferencia. Con relación a esa cuestión, sostendré que, si bien la diferencia entre estas dos concepciones puede tener algún interés filosófico (similar a aquel suscitado por el debate entre causalistas y estadisticalistas en el contexto de la filosofía de la biología evolutiva; Sober 1984; Matthen y Ariew 2002; Walsh et al. 2002), queda para demostrar que tenga algún significado empírico.

En un sentido trivial, la genética de poblaciones y la epidemiología son teorías diferentes porque tienen dos objetos diferentes. La genética de poblaciones tiene como objetivo el de rastrear el cambio en las distribuciones genotípicas (o fenotípicas, en el caso de la genética cuantitativa), mientras que la epidemiología estudia la difusión de enfermedades infecciosas. Dada las diferencias entre los dos fenómenos, las dos teorías adoptan modelos matemáticos propios. Sin embargo, ambas teorías se dedican a estudiar fenómenos relacionados con dinámicas de poblaciones. Además, como observado por los biólogos Steve Peterson y Mark Viney, "hay [...] una clara analogía entre herencia - la transmisión de genes de una generación a otra - e infección — la transmisión de 
parásitos de un huésped a otro-y las consecuencias de ambos procesos en el nivel de las poblaciones" (Peterson y Viney 2000, 528). Si admitimos esa analogía, lo que distingue la genética de poblaciones y la epidemiología sería más el enfoque que cada una de estas disciplinas adopta respecto a fenómenos parecidos. Para Peterson y Viney, la genética de poblaciones estudiaría los fenómenos de transmisión directamente en el nivel de las poblaciones, mientras que la epidemiología se dedicaría a dar cuenta de la emergencia de patrones poblacionales a partir de agregados de instancias de transmisión.

En ese sentido, los epidemiólogos culturales identifican una diferencia real entre las dos disciplinas. Lo que está en cuestión, sin embargo, no es sólo si tal diferencia existe, sino si tiene las implicaciones que Sperber y los demás parisinos le atribuyen. Para soportar su postura "separatista", los parisinos necesitarían mostrar que los resultados obtenidos a partir de modelos de genética de poblaciones son sustancialmente divergentes de los resultados obtenidos a partir de modelos epidemiológicos. Es con relación a ese aspecto que, por lo que puedo ver, los parisinos fallan en ofrecer razones convincentes. Que los modelos de las dos teorías en cuestión sean compatibles, o incluso equivalentes, es algo que ya sostenían, casi 40 años atrás (en la "prehistoria" de la teoría de la doble herencia), los genetistas Luca Luigi Cavalli-Sforza y Marcus Feldman. En su clásico Cultural Transmission and Evolution: A Quantitative Approach, los autores notan:

La analogía de una enfermedad infecciosa con la difusión de una innovación en el ámbito de una generación es tal vez más acertada que aquella con una mutación exitosa. Sin embargo, las teorías matemáticas formulada por cualquier una de las tres variables número de individuos portadores de una enfermedad infecciosa, la proporción portadora de mutaciones ventajosas, o el número de individuos que han adoptado una innovación - son formalmente idénticas cuando estudiadas a este nivel elementar. (Cavalli-Sforza y Feldman 1981, 33; cursivo en el original)

Una opinión parecida tiene Aunger (2001), el cual imputa las diferencias formales entre genética de poblaciones y epidemiología más a factores contingentes debidos a la historia y a la agenda de cada disciplina que a reales diferencias conceptuales. La epidemiología, con su énfasis en la relación entre los agregados de individuos y las consecuencias poblacionales de las interacciones entre estos, privilegia un análisis espacial de las dinámicas de difusión. Para eso, recurre a varias herramientas formales derivadas de la teoría de redes - network theory - (Peterson y Viney 2000, 530), cuya relevancia no sea tal vez evidente para la genética de poblaciones. Por otro lado, la genética de poblaciones se enfoca sobre la dimensión temporal de los fenómenos de difusión. Es, plausiblemente, en virtud de tal enfoque diferente que los genetistas de poblaciones necesitan realizar una mayor abstracción con respecto al nivel de los individuos y concentrarse exclusivamente en el nivel de las poblaciones.

Lo que emerge de esta conceptualización es que, con respecto a un cierto dominio de fenómenos de distribución de variantes en una población, la genética de poblaciones y

Revista de Humanidades de Valparaíso, 2019, No 14, 223-240 
la epidemiología son dos disciplinas complementarias, y en ningún sentido en conflicto. En el contexto de una teoría de la evolución cultural, tal complementariedad podría ser entendida de la siguiente manera. Por un lado, tendríamos estudios de carácter epidemiológico, que conectan lo que sabemos sobre los sesgos humanos de aprendizaje social con la probable difusión espacial de una representación/variable cultural. La noción de atractor tendría, de acuerdo con lo que ha sido sostenido por varios epidemiólogos culturales (Buskell 2017b; Scott-Phillips et al. 2018), la función de monitorear los efectos agregativos de las interacciones culturales. La información obtenida a partir de estos estudios epidemiológicos constituiría una importante fuente para construir, por otro lado, modelos temporales de acumulación y cambio cultural, los cuales podrían beneficiarse de herramientas formales derivadas de la genética de poblaciones (como han sostenido los teóricos de la doble herencia).

Es interesante notar que, aunque nominalmente poco atraídos por tal proyecto compatibilista, algunos epidemiólogos culturales ya lo promueven tácitamente. Es el caso, por ejemplo, de Claidière et al. (2014). En ese artículo, los autores critican la teoría de la doble herencia etiquetándola como "seleccionista". De su punto de vista, el fallo de esa teoría sería que insiste demasiado sobre la adaptividad, genética o cultural, de las variantes culturales más difusas en una sociedad. En otras palabras, la teoría de la doble herencia sería culpable de una especie de "panadaptacionismo cultural". En contra de tal posición, Claidière y sus colaboradores elaboran un proto-modelo matemático - las "matrices evolutivas causales" - que, a partir de una concepción no-replicativa de la información cultural, permitiría inferir procesos de distribuciones de representaciones culturales. El resultado es que, en la mayoría de escenarios, las distribuciones de representaciones culturales no coincidirían con las distribuciones previstas por un enfoque seleccionista.

Lo que Claidière y sus colaboradores parecen ocultar al lector es que el empleo de herramientas conceptuales derivadas de la genética de poblaciones no implica la adhesión a una perspectiva seleccionista. De hecho, los modelos de genética de poblaciones están explícitamente formulados para tomar en cuenta otras causas de los procesos evolutivos además que la selección natural, tales como deriva genética, mutación, migración etc. Es más, en la medida en que las matrices evolutivas causales son modelos que tienen como objetivo el de rastrear frecuencias de representaciones culturales - haciendo completa abstracción de como tales frecuencias son realizadas por agregados de interacciones individuales - ellas mismas podrían ser interpretadas como modelos derivados de la genética de poblaciones. Y no habría nada de lo que avergonzarse: la epidemiología tiene que dejar paso a la genética de poblaciones cuando se trata de observar una población "desde arriba”, para entender dinámicas de más larga escala y duración. 
París, California y la búsqueda por una teoría del cambio cultural

Lorenzo Baravalle

\section{Agregados y causas a nivel de poblaciones: el interés filosófico del debate entre parisinos y californianos}

Llegados a este punto, los simpatizantes de la epidemiología cultural podrían objetar que lo que he discutido en la sección anterior es irrelevante. Lo que está en discusión cuando se comparan el papel de la epidemiología y de la genética de poblaciones en el contexto de una teoría de la evolución cultural no es el uso de ciertas herramientas formales, sino que dos diferentes concepciones causales del cambio cultural. Como vimos en las secciones 2 y 3, parecería que la analogía entre transmisión cultural y herencia genética compromete los teóricos de la doble herencia con un cierto realismo con relación a causas actuando en un nivel sobre-individual (las presiones selectivas culturales y otros tipos de causas evolutivas). La analogía con la epidemiología, en cambio, dispensaría los parisinos de tal compromiso: de un punto de vista causal, todas las dinámicas evolutivas culturales son reducibles a interacciones individuales. Como dijimos, eso sería más naturalísticamente aceptable y constituiría una razón de peso para inclinarse a favor de la perspectiva epidemiológica (véase, por ejemplo, Scott-Phillips et al. 2018).

El problema que veo con esa estrategia argumentativa es que promete más de lo que puede ofrecer. Es cierto que la caracterización causal de los procesos de acumulación y cambio cultural son diferentes en la teoría de la doble herencia y en la epidemiología cultural. Sin embargo, no queda claro si tal diferencia tiene alguna significativa consecuencia testable o si — se me perdone la paráfrasis de una célebre expresión - es una diferencia que no hace (por lo menos empíricamente) ninguna diferencia.

¿Qué tipo de compromiso ontológico asumen los teóricos de la doble herencia cuando afirman que la transmisión cultural es un "canal" de herencia y que las variantes culturales están sujetas a presiones selectivas (o a otras fuerzas evolutivas; Baravalle 2019)? Para poder responder a esta pregunta, debemos antes entender mejor que tipo de relaciones causales sobre-individuales suelen ser postuladas en el contexto del estudio evolutivo de poblaciones en biología. Cuando un genetista de poblaciones dice que un cierto rasgo fue seleccionado (o evolucionó por deriva, o fue introducido por procesos migratorios etc.), lo que quiere decir es que un cierto conjunto de condiciones ambientales, bióticas y/o sociales hizo que la frecuencia de tal rasgo se incrementara en la población. Por supuesto, para que eso pueda ocurrir, es necesario que los individuos que componen la población se comporten de cierta manera: algunos morirán sin tener descendencia, mientras que otros serán muy prolíficos. Sin embargo, podría argumentarse, el proceso evolutivo no es simplemente el agregado de tales circunstancias individuales. Autores como Elliott Sober (1980; Shapiro y Sober 2007) o Roberta Millstein (2006) han insistido sobre el hecho de que lo que cambia en el proceso evolutivo - esto es, frecuencias de rasgos u otros aspectos demográficos, como la estructura démica de la población- son propiedades de poblaciones, no reducibles a propiedades de individuos. Por esa razón, es más correcto decir que es la población como un todo (y no un agregado de individuos) la que establece ciertas dinámicas causales con el ambiente, bioma, o contexto social.

Revista de Humanidades de Valparaíso, 2019, No 14, 223-240

(c) $(1)(9)$ CC BY-NC-ND 
Cuando los teóricos de la doble herencia comparan las variantes culturales a rasgos fenotípicos sujetos a factores evolutivos análogos a aquellos que influyen en las distribuciones de los rasgos fisiológicos o morfológicos, lo que plausiblemente quieren decir es que, a lo largo de las cadenas de transmisión culturales, actúan factores que, globalmente, producen cambios en la composición de la población. Como las dinámicas evolutivas dependientes de la herencia genética, también las dinámicas evolutivas culturales necesitan ser realizadas por individuos que interactúan de cierta manera. Sin embargo, como también en el caso de los procesos evolutivos dependientes de la herencia genética, puede sostenerse que el proceso evolutivo cultural no es solamente consecuencia del agregado de tales interacciones. Las frecuencias cambiantes de rasgos culturales, la estructura démica de la población y otras características sociales e institucionales de una población son todas propiedades de la población, no directamente reducibles a propiedades de sus miembros. Sería, por lo tanto, correcto considerar el proceso evolutivo cultural como un proceso causal en el nivel de la población.

En el contexto del debate filosófico sobre la estructura causal y explicativa de la teoría evolutiva en biología no han faltado los que han criticado la perspectiva presentada aquí arriba. Matthen y Ariew (2002) y Walsh et al. (2002) - los llamados estadisticalistashan objetado - en contra de la tesis llamada de causalista - que no es necesario postular un nivel de interacciones causales sobre-individuales para dar cuenta del lenguaje de los biólogos evolutivos. Desde el punto de vista de estos filósofos, los conceptos comúnmente usados para referirse a procesos evolutivos - como selección, deriva, migración etc.no se refieren a causas, sino que son solamente artefactos estadísticos. La única cosa que propiamente "existe" son individuos naciendo, interactuando de cierta manera, teniendo una mayor o menor descendencia y, finalmente, muriendo. Solo en ese nivel hay relaciones causales. El problema es que sería imposible, para un biólogo evolutivo, rastrear cada uno de estos individuos, dentro de una población y por varias generaciones, para entender como la composición de la población cambia. Es aquí donde intervienen los conceptos de selección, deriva etc.: ellos funcionan como instrumentos para "mantener la cuenta" de los nacimientos, reproducciones y muertes y, de esa manera, hacernos entender mejor las causas del cambio evolutivo. Sin embargo, estos conceptos no denotan ningún proceso "real", son constructos resultantes de la teoría; un poco como la "expectativa de vida" de la que hablan las aseguradoras.

Ahora, independientemente de las simpatías de cada uno, lo que en realidad está en cuestión en el debate entre causalistas y estadisticalistas - como ha notado justamente Glennan (2008) — son dos diferentes nociones de causa. Por un lado, los causalistas hacen referencia a una noción de causa como relevancia causal; por el otro, los estadisticalistas se refieren a una noción de causa como producción. La noción de relevancia causal (como es discutida, por ejemplo, en Woodward 2003) tiene que ver con la idea de que una causa es un difference maker, es decir, un evento o una propiedad que, de haber sido diferente, el efecto también habría sido diferente. En ese sentido, cuando un biólogo dice 
que la predominancia de una cierta variante mimética en una población de polillas es debida a la presión selectiva creada por las fábricas contaminantes limítrofes a su hábitat (como en el célebre caso de Biston betularia), expresándose así en términos causales poblacionales, lo que quiere decir es lo siguiente: ceteris paribus, si no hubiese habido fábricas contaminantes, las frecuencias de variantes se habrían mantenido constantes. Esa manera de referirse a causas, correcta y común en las ciencias especiales, no excluye otro uso de la noción de causa como producción (derivado de Salmon 1998; Dowe 2000), que hace referencia a las circunstancias individuales que han realizado y hecho posible el proceso poblacional.

De mi punto de vista, el énfasis puesto por los epidemiólogos culturales sobre el aspecto agregativo de las dinámicas culturales, si bien puede enriquecer conceptualmente la teoría de la evolución cultural, no pasa de tener un valor principalmente filosófico, similar a aquel de la interpretación estadisticalista de la biología evolutiva. Tanto los estadisticalistas como los parisinos son escépticos con respecto a la posibilidad de concebir procesos poblacionales de cambio evolutivo como dotados de características causales propias. Ambos sostienen la necesidad, para una caracterización más naturalísticamente aceptable de los procesos evolutivos, de descargar el peso de la descripción causal de tales procesos sobre los individuos que los realizan. Sin embargo, puede argumentarse, en contra de los parisinos, que su perspectiva, al igual que la de los estadisticalistas, es compatible con aquella de los que atribuyen un papel causal a los procesos evolutivos en el nivel de las poblaciones. Así como el desacuerdo entre causalistas y estatisticalistas puede ser imputado a diferentes interpretaciones — ambas absolutamente legítimas - de las nociones causales involucradas en la caracterización de los procesos evolutivos, algo análogo podría decirse con relación al desacuerdo entre parisinos y californianos.

Dicho esto, la cosa aquí más importante es que, tanto en el caso del debate causalistas/ estadisticalistas como en el presente caso, los científicos llamados a construir modelos evolutivos pueden tranquilamente ignorar la cuestión. Esta no influye directamente sobre cómo los modelos son construidos: es "empíricamente opaca", por decirlo de alguna manera. Claramente, los modelistas pueden elegir estrategias más agregativas o, alternativamente, centradas en propiedades de poblaciones (Illari y Russo 2014, cap. 5). Pero eso no implica la adopción, por parte de los modelistas, de presupuestos ontológicamente profundos sobre donde las causas de los procesos evolutivas están "realmente". En la practica, la elección de un modelo "realista" con respecto a causas al nivel de poblaciones, derivado de la genética de poblaciones, u otro "deflacionario", derivado de la epidemiología, depende de consideraciones instrumentales, similares a aquellas discutidas en la sección anterior. De hecho, en el contexto de la teoría de la evolución cultural, trabajos como el realizado por El Mouden et al. (2014), elaborado por autores cercanos a la epidemiología cultural, o incluso el proto-modelo de las matrices causales del que ya hemos hablado previamente (Claidière et al. 2014) podrían haber sido realizados por teóricos de la doble herencia. 
Para poder soportar las pretensiones de secesión de la teoría de la doble herencia, los epidemiólogos culturales deberían presentar evidencia de que los diferentes mecanismos de transmisión cultural postulados por las dos teorías tienen efectos poblacionales diferentes; $\mathrm{o}$, mejor aún, que las dinámicas evolutivas culturales reales pueden ser descritas y explicadas por el arsenal teórico de la epidemiología cultural, pero no con el de la teoría de la doble herencia. Desafortunadamente para ellos, no creo que los epidemiólogos culturales hayan conseguido hacer eso. En parte eso se debe, sin duda, al hecho de que más allá del énfasis sobre la analogía con la epidemiología y sobre la teoría de los atractores, muy pocos modelos matemáticos han empleado operacionalmente estos conceptos como admitido por los mismos epidemiólogos culturales- (Scott-Phillips et al. 2018, 171). Sin modelos de este tipo, es difícil corroborar si las consecuencias poblacionales previstas por la teoría epidemiológica son o no compatibles con las previstas por la teoría de la doble herencia. Más allá de eso, sin embargo, sospecho que hay una dificultad de principio en la realización de las ambiciones de los parisinos. Aunque estos estén en lo cierto con respecto a los mecanismos de transmisión cultural, dudo - por las razones esbozadas en la sección anterior- de que una teoría análoga a la genética de poblaciones como es la teoría de la doble herencia sería incapaz de adaptarse para dar cuenta de las consecuencias poblacionales de tales mecanismos de transmisión.

\section{Conclusión}

A lo largo de este artículo, mi objetivo ha sido el de mostrar que las dos principales propuestas teóricas en el contexto de los estudios sobre evolución cultural, esto es, la teoría de la doble herencia y la epidemiología cultural, son más compatibles de lo que a los epidemiólogos culturales les gustaría admitir. Mi argumento ha sido básicamente el siguiente. Dos tipos de consideración podrían justificar la interpretación de la teoría de la doble herencia y la epidemiología cultural como teorías por lo menos parcialmente incompatibles. El primero es de carácter formal. Por un lado, la teoría de la doble herencia se basa, en buena medida, sobre una analogía con la genética de poblaciones y emplea modelos derivados de esa disciplina. Por el otro, la epidemiología cultural (como el mismo nombre deja claro) se inspira en una analogía con la epidemiología. Si, por lo tanto, resultase que la genética de poblaciones y la epidemiología son teorías en cierto grado incompatibles, eso podría tener consecuencias también en el contexto de la teoría de la evolución cultural. Sin embargo, he mostrado en la sección 4 que ese no es el caso. La genética de poblaciones y la epidemiología son teorías formalmente compatibles, y hasta complementarias en algunas de sus aplicaciones.

El segundo aspecto que podría justificar una nítida demarcación entre epidemiología cultural y teoría de la doble herencia es de carácter causal. Las analogías con la epidemiología y con la genética de poblaciones parecen soportar dos distintas concepciones de la estructura causal de las dinámicas poblacionales: meramente agregativa en el caso de la

Revista de Humanidades de Valparaíso, 2019, No 14, 223-240 
epidemiología y realista con respecto a causas sobre-individuales en el caso de la genética de poblaciones. Si bien esta caracterización de diferentes concepciones de la estructura causal de las dinámicas poblacionales revela un rasgo genuino de la controversia entre epidemiólogos culturales y teóricos de la doble herencia, he argumentado en la sección 5 que se trata de una divergencia más filosófica que científica (esto es, relacionada con explicaciones o predicciones empíricamente testables).

Para concluir, me gustaría aclarar que mi argumento no tiene, en absoluto, el objetivo de disminuir el valor de los trabajos realizados en el contexto de la tradición epidemiológica. Por el contrario, es justamente porque creo que estos trabajos contribuyen, con una perspectiva original, al entendimiento de las dinámicas de acumulación y cambio cultural que insisto sobre la necesidad de integrarlos con los trabajos realizados por los teóricos de la doble herencia. Como Scott-Phillips y sus colaboradores reconocen:

un foco excesivo sobre el contraste entre estos enfoques sería contraproductivo. Hay un equilibrio a ser alcanzado aquí, entre la elucidación de genuinos malentendidos por un lado, y, por el otro, la apariencia de una pelea miope entre especialistas - una consecuencia que deseamos evitar. $(2018,168)$

En la práctica, sin embargo, esa consecuencia no siempre se ha conseguido evitar. No queda que esperar que, en futuro, haya mayor entendimiento y colaboración.

\section{Agradecimientos}

Esta investigación ha sido financiada por el Conselho Nacional de Desenvolvimento Científico e Tecnológico de Brasil (CNPq - beca $\left.n^{\circ} 402619 / 2016-1\right)$ y por el Fondo Nacional de Desarrollo Científico y Tecnológico de Chile (FONDECYT REGULAR - beca $\left.n^{\circ} 1171017\right)$.

\section{Referencias bibliográficas}

Acerbi, A., Mesoudi, A. (2015). If we are all cultural Darwinians what's the fuss about? Clarifying recent disagreements in the field of cultural evolution. Biology \& Philosophy, 30(4): 481503. doi: 10.1007/s10539-015-9490-2

Aunger, R. (2001). Introduction. En R. Aunger (ed.), Darwinizing culture: The status of Memetics as a science, pp. 1-23. Oxford: Oxford University Press.

Baravalle, L. (2019). Cultural evolutionary theory as a theory of forces. Synthese, online-first. doi: 10.1007/s11229-019-02247-0

Boyd, R., Richerson, P. (1985). Culture and the evolutionary process. Chicago: The University of Chicago Press. 
Buskell, A. (2017a). What are cultural attractors? Biology \& Philosophy, 32(3): 377-394. doi: $10.1007 / \mathrm{s} 10539-017-9570-6$

Buskell, A. (2017b). Cultural Attractor Theory and explanation. Philosophy, Theory, and Practice in Biology, 13(9). doi: 10.3998/ptb.6959004.0009.013

Cavalli-Sforza, L. L., Feldman, M. (1981). Cultural Transmission and Evolution: a Quantitative Approach. Princeton (NJ): Princeton University Press.

Claidière, N., Scott-Phillips, T. C., Sperber, D. (2014). How Darwinian is Cultural Evolution? Philosophical Transactions of the Royal Society B, 369. doi: 10.1098/rstb.2013.0368

Dowe, Phil (2000). Physical Causation. Cambridge: Cambridge University Press.

El Mouden, C., André, J.-B., Morin, O., Nettle, D. (2014). Cultural transmission and the evolution of human behaviour: a general approach based on the Price equation. Journal of Evolutionary Biology, 27: 231-241. doi: 10.1111/jeb.12296

Glennan, S. (2008). Productivity, relevance and natural selection. Biology \& Philosophy, 24: 32539. doi: 10.1007/s10539-008-9137-7

Illari, P., Russo, F. (2014). Causality. Philosophical Theory meets Scientific Practice. Oxford: Oxford University Press.

Kendal, R. L., Boogert, N. J., Rendell, L., Laland, K. N., Webster, M., Jones, P. L. (2018). Social learning strategies: Bridge-building between fields. Trends in Cognitive Science, 22(7): P651-P665. doi: 10.1016/j.tics.2018.04.003

Matthen, M., Ariew, A. (2002). Two Ways of Thinking about Fitness and Natural Selection. Journal of Philosophy, 99(2): 55-83. doi: 10.2307/3655552

Mesoudi, A. (2011). Cultural Evolution: How Darwinian Theory Can Explain Human Culture and Synthesize the Social Sciences. Chicago: The University of Chicago Press.

Millstein, R. (2006). Natural Selection as a population-level causal process. British Journal for the Philosophy of Science, 57(4): 627-53. URL: www.jstor.org/stable/4489089

Morin, O. (2016a). Reasons to be fussy about cultural evolution. Biology \& Philosophy, 31: 44758. doi: 10.1007/s10539-016-9516-4

Morin, O. (2016b). How traditions live and die. Oxford: Oxford University Press.

Peterson, S., Viney, M. E. (2000). The interface between epidemiology and population genetics. Parasitology today, 16(12): 528-532. doi: 10.1016/S0169-4758(00)01776-2

Richerson, P. J. (2017). Recent critiques of dual inheritance theory. ESIC, 1, 203-11. URL: http:// www.des.ucdavis.edu/faculty/Richerson/Morin\%20Lewens\%20formatted\%20final.pdf

Richerson, P., Boyd, R. (2005). Not by Genes Alone: How Culture Transformed Human Evolution. Chicago: The University of Chicago Press.

Salmon, W. (1998). Causality and explanation. Oxford: Oxford University Press. 
Scott-Phillips, T., Blancke, S., Heintz, C. (2018). Four misunderstandings about cultural attraction. Evolutionary Anthropology, 27: 162-73. doi: 10.1002/evan.21716

Shapiro, L., Sober, E. (2005). Epiphenomenalism - The Do's and the Don'ts. En G. Wolters, P. Machamer (eds.), Thinking about causes: From greek philosophy to modern physics, pp. 235-264. Pittsburg: University of Pittsburg Press.

Sober, E. (1980). Evolution, population thinking, and essentialism. Philosophy of Science, 47(3): 350-383. URL: http://www.jstor.org/stable/186950

Sober, E. (1984). The Nature of Selection. Chicago: The Chicago University Press.

Sperber, D. (1996). Explaining culture. A naturalistic approach. Oxford: Blackwell.

Sperber, D. (2001). An Objection to the memetic approach to Culture. En R. Aunger (ed.) Darwinizing culture: The status of Memetics as a science, pp. 163-174. Oxford: Oxford University Press.

Sperber, D., Claidière, N. (2008). Defining and explaining culture (comments on Richerson and Boyd, Not by genes alone). Biology \& Philosophy, 23: 283-292. URL: http://www. dan.sperber.fr/wp-content/uploads/2008/09/NotByGenesAloneCommentary-SperberClaidiere.pdf

Sterelny, K. (2017). Cultural evolution in California and Paris. Studies in History and Philosophy of Biology and Biomedical Sciences, 62: 42-50. doi: 10.1016/j.shpsc.2016.12.005

Tooby, J., Cosmides, L. (1992). The Psychological Foundations of Culture. En J. Barkow, L. Cosmides, J. Tooby (eds.), The Adapted Mind: Evolutionary Psychology and the Generation of Culture, pp. 19-136. New York: Oxford University Press.

Walsh, D., Lewens T., Ariew A. (2002). The trials of life: Natural selection and random drift. Philosophy of Science, 69(3): 452-73. doi: 10.1086/342454

Woodward, J. (2003). Making things happen. A theory of causal explanation. Oxford: Oxford University Press. 